\section{Early perioperative management of bile duct injuries acquired during laparoscopic cholecystectomy}

With the advent of laparoscopic technology, open cholecystectomy has been supplanted by laparoscopic cholecystectomy for the treatment of calculous biliary disease. Unfortunately, this has led to an increase in the incidence of major bile duct injuries (BDI). Several studies have evaluated the long-term management of patients with BDI but few have focused on their early perioperative management. Sicklick et al. prospectively studied 200 patients and analyzed the perioperative management of $\mathrm{BDI}$ following laparoscopic cholecystectomy.

The mean time from BDI to referral was 29.1 weeks, although this was variable depending upon patients' presentations. Twenty-five patients did not undergo surgical repair, including three who died because of uncontrolled sepsis after delayed referral. The remaining 22 patients underwent successful balloon dilation of an anastomotic stricture. Of the 175 patients that underwent biliary reconstruction, 172 had hepaticojejunostomies and 3 had end-to-end ductal repairs. Seventy-five patients had at least one postoperative complication, although all were managed nonoperatively. There were three deaths in the postoperative period.

In this study, the timing of the operation, presenting symptoms and history of previous attempts at BDI repair did not affect the incidence of the most common perioperative complications or the postoperative length of stay. The authors note that control of sepsis and bile leak are the primary goals of initial BDI management, whereas reconstruction can be carried out when the inflammation has subsided. This study supports the importance of early referral to a tertiary care hospital and definitive biliary reconstruction by an experienced hepatobiliary surgeon.

Original article Sicklick JK et al. (2005) Surgical management of bile duct injuries sustained during laparoscopic cholecystectomy: perioperative results in 200 patients. Ann Surg 241: 786-792

\section{Free radical damage in gastroesophageal reflux disease}

The severity of Barrett's esophagus and reflux esophagitis is not determined solely by acid exposure. Studies suggest an involvement of free radicals in the associated damage to the esophageal mucosa but the protective role of antioxidant defense mechanisms in such reflux diseases remains unclear. A recent study by Jiménez and colleagues thus investigated the profile and balance between free radicals and antioxidant systems in Barrett's esophagus and esophagitis.

In this study, esophageal biopsies were taken from 101 consecutive patients with gastroesophageal reflux disease (GERD) attending the Endoscopy Unit of the University Hospital of Zaragoza, and from 28 controls with no clinical symptoms of GERD. Levels of reactive oxygen and nitrogen species were then analyzed and antioxidant status determined.

The results demonstrated that, compared with controls, patients with erosive esophagitis and Barrett's esophagus had significantly higher superoxide anion levels, and the generation of peroxynitrate was greater in Barrett's esophagus compared with esophagitis patients and controls. The activity of superoxide dismutase (SOD) antioxidant was decreased and that of glutathione and catalase increased in GERD patients compared with controls. To determine the mechanism by which SOD levels were decreased, Cu,ZnSOD and MnSOD protein expression was evaluated. Both were found to increase with lesion severity and to be highest in patients with Barrett's esophagus.

The authors concluded that " $\ldots$.. decrease in SOD antioxidant activity leading to increased mucosal levels of superoxide anion and peroxynitrate radicals may contribute to the development of esophageal damage and Barrett's esophagus in patients with GERD." It was thus suggested that SOD administration might be implicated as a therapeutic target to treat such conditions.

Original article Jiménez P et al. (2005) Free radicals and antioxidant systems in reflux esophagitis and Barrett's esophagus. World J Gastroenterol 11:2697-2703

\section{Prophylactic anti-HBV therapy in hematopoietic stem-cell transplantation}

Exclusion of hepatitis B virus surface antigen (HBsAg)-positive donors would greatly limit the 